\title{
Alcances y limitaciones de la ciencia abierta en Latinoamérica: análisis de las políticas públicas y publicaciones científicas de la región
}

\author{
Daniela De Filippo \\ Universidad Carlos III de Madrid \\ dfilippo@bib.uc3m.es \\ https://orcid.org/0000-0001-9297-9970
}

\author{
Maria Guillermina D'Onofrio \\ Ministerio de Educación, Cultura, Ciencia y Tecnología (MECCYT), Argentina \\ mgdonofrio@mincyt.gob.ar \\ https://orcid.org/0000-0002-5896-5193
}

\section{Scope and limitations of open science in Latin America: analysis of public policies and scientific publications}

\section{RESUMEN ABSTRACT}

Este trabajo analiza las iniciativas sobre Ciencia Abierta en Latinoamérica a través del estudio cualitativo exploratorio de las políticas públicas y cuantitativo cientométrico de las publicaciones científicas. Para ello se han consultado fuentes de organismos oficiales y se ha usado la base de datos internacional SCOPUS para la obtención de los principales indicadores bibliométricos. Los resultados obtenidos muestran que es en la última década cuando empiezan a surgir iniciativas en este tema y, en concreto, en 7 países: Argentina, Brasil, Chile, Colombia, México, Perú y Uruguay. La mayor parte de las políticas se orientan a la promoción de infraestructuras para el acceso abierto, fundamentalmente a publicaciones. La producción científica sobre ciencia abierta se concentra en los últimos 5 años y la región va aumentando sus aportes al mundo. Destacan los temas de acceso abierto, datos abiertos, innovación en investigación e investigación participativa pero no hay una tendencia correlativa

a su publicación en revistas científicas de acceso abierto. Aunque se ha hecho evidente el interés por la ciencia abierta en un sentido amplio a nivel académico, por el momento gran parte de las iniciativas a nivel de políticas y legislación se han centrado en el acceso abierto.

PALABRAS CLAVE

Ciencia abierta; Acceso abierto; Latinoamérica; Bibliometría; Políticas públicas
This paper analyzes the Open Science initiatives in Latin America through the study of public policy and scholarly publications. Sources from governmental agencies have been consulted and the international database SCOPUS has been exploited to obtain the main bibliometric indicators. The results show that it is in the last decade when initiatives in this subject begin to emerge, specifically in 7 countries: Argentina, Brazil, Chile, Colombia, México, Peru and Uruguay. Most of the policies are oriented towards the promotion of infrastructures for open access. Publications on open science are concentrated in the last 5 years and the region is increasing its contribution to the world. The topics of Open Access, Open Data, research innovation and participative research stand out but there is no tendency to publish these topics in open access journals. Although interest in open science has become evident, much of the policy and legislative initiatives have focused on open access.

KEYWORDS

Open science; Open access; Latin America;

Bibliometrics; Public policies

De Filippo, D. y D'Onofrio, M.G. (2019). Alcances y limitaciones de la ciencia abierta en Latinoamérica: análisis de las políticas públicas y publicaciones científicas de la región. Hipertext.net, (19), 32-48. DOI:10.31009/hipertext.net.2019.i19.03 


\section{Introducción}

Desde hace poco más de una década, el movimiento de ciencia abierta ha tenido un impacto notable en el desarrollo de las actividades científicas. Aunque son muchas las definiciones que podemos encontrar, sin dudas una de las más extendidas es la que la concibe como una práctica científica en la cual otros pueden colaborar y contribuir, donde los datos de investigación, las notas de laboratorio y los diferentes procesos de investigación estén disponibles libremente y pueden ser reutilizados, redistribuidos y reproducidos (Foster, 2016). En pocas palabras, la ciencia abierta es un conocimiento transparente y accesible que se comparte y se desarrolla a través de redes de colaboración (Vicente-Sáez y Martínez-Fuentes 2018).

Sin dudas, las nuevas tecnologías, la producción masiva de datos, las redes sociales científicas, la ciencia ciudadana, los recursos educacionales y el código abierto, en conjunto, han generado una forma diferente de hacer, pensar y difundir la ciencia. Tal como mencionan algunos autores "la ciencia abierta es un cambio de paradigma en la manera de hacer ciencia. Ésta no cambia sustancialmente con respecto a sus motivaciones y objetivos, pero sí lo hace (sustancialmente) en cuanto a sus métodos. El cambio no está en lo que se hace, sino en cómo se hace" (Anglada y Abadal, 2018). Es por eso por lo que mucho se habla del "cambio de paradigma" que representa la ciencia abierta, un cambio cultural en la forma en que las partes interesadas en la investigación, la educación y el intercambio de conocimientos, crean, almacenan, comparten y entregan los resultados de su actividad (LERU, 2018).

Aunque los orígenes del proceso de apertura del conocimiento científico pueden rastrearse más de cuarenta años atrás, es hacia los años 2002 y 2003 cuando se establece el concepto de acceso abierto con las declaraciones de Budapest, Bethesda y Berlín (Palma Peña, 2019), y se va consolidando un movimiento de progresiva apertura de la literatura científica financiada con fondos públicos.

Si bien es posible pensar que el acceso abierto se ha convertido en el abanderado de la ciencia abierta, es evidente que el concepto va más allá. Es, en definitiva, un cambio de sistema, donde la ciencia se lleva a cabo y se comunica de una manera que permite a distintos actores sociales contribuir y sumar al esfuerzo de investigación, con todo tipo de datos, resultados y protocolos libremente disponibles, en diferentes etapas del proceso de investigación (Research Information Network, 2010).

Para incentivar la adopción de prácticas de ciencia abierta, en los últimos años diferentes organismos internacionales, regionales y nacionales han desarrollado numerosas políticas y estrategias para su promoción y consolidación (Santos et al,
2017).

En este sentido, la Unión Europea ha sido uno de los grandes promotores mundiales y, tal como mencionan Anglada y Abadal (2018), las motivaciones para el impulso de la ciencia abierta han sido, principalmente, de dos tipos: político-sociales y científicas. Las primeras se basan en la necesidad de Europa de mantener y aumentar los niveles de bienestar, para lo que se necesita una economía fuerte que debe tener su centro en la innovación derivada de la investigación científica. Con respecto a las motivaciones científicas, los autores afirman que el movimiento de la ciencia abierta quiere adecuar la comunicación científica a las posibilidades tecnológicas actuales y aumentar su eficacia. Su trayectoria comienza en 2012 con la adopción progresiva del acceso abierto como principio rector de su política científica. Desde entonces, cada vez se ha hecho más presión -y se ha generado más conciencia- para que los proyectos financiados en el marco de los Programas Europeos (y en concreto del Programa Horizonte 2020), pongan en abierto toda la literatura científica producida en ellos (European Union, 2013). Otras iniciativas, promovidas también por la Unión Europea, son un reflejo del interés de este organismo de coordinación de políticas regional por la expansión y consolidación de la ciencia abierta. Entre ellas es posible mencionar los avances en el campo de las infraestructuras y repositorios digitales abiertos con el Proyecto OpenAire que permite la agregación de toda la literatura científica producida en proyectos bajo financiación europea y proporciona vínculos entre esas publicaciones y los datos subyacentes. A esto se suman también la European Open Science Cloud (EOSC) y el Open Science Monitor (European Commission, 2016; 2017).

Siguiendo las directrices regionales, algunos países europeos también han desarrollado políticas nacionales para promover y expandir la ciencia abierta (Fosci et al, 2019). Entre algunos de los ejemplos más destacados, es posible mencionar el caso de Finlandia con la Open Science and Research Iniciative patrocinada por el Ministerio de Educación y Cultura (Finland, 2014; Forsström y Haataja, 2016); o el de Holanda con el National Plan Open Science (Netherlands, 2017). En la península Ibérica, el Ministerio de Ciencia, Tecnología y Educación Superior de Portugal estableció como meta para el año 2018 la adopción y aplicación de una política científica abierta, definiendo los principios de aplicación en cuatro ejes: acceso abierto y datos abiertos; infraestructuras y preservación digital; evaluación científica; y responsabilidad social científica (Portugal, 2016; da Silva, 2017). En España, el Plan Estatal de Investigación Científica y Técnica y de Innovación 2017-2020 definió la apertura de resultados y datos de investigación obtenidos bajo financiación pública como obligatoria (España, 2017).

Más allá de las fronteras europeas, también se encuentran ejemplos como el de Canadá, que ha implementado el Action Plan en Open Government 2.0 permitiendo mayor acceso a los resultados de investigación científica financiada con fondos 
públicos y facilitando una mayor colaboración entre el sector público y el privado (OECD, 2015). Por su parte, en Sudáfrica se ha realizado un esfuerzo destacado para el desarrollo de políticas de acceso abierto y la promoción de la apertura científica a través del impulso a los repositorios (UNESCO, 2015).

En el caso concreto de Latinoamérica, el movimiento de ciencia abierta se hizo más evidente de la mano de la apertura de las publicaciones científicas. Una de las iniciativas más destacadas es, precisamente, "LA Referencia" (Red Federada de Repositorios Institucionales de Publicaciones Científicas) creada en 2012, y cuya experiencia se basa en acuerdos técnicos y organizativos entre organismos públicos de ciencia y tecnología para apoyar las estrategias nacionales de acceso abierto en América Latina mediante una plataforma interoperable. Actualmente está integrada por 10 países (Argentina, Brasil, Chile, Colombia, Costa Rica, Ecuador, El Salvador, México, Perú y Uruguay), participa en COAR (Confederation of Open Access Repositories) y colabora con OpenAire (LA Referencia, 2019).

Recientemente, el Foro Abierto de Ciencias de América Latina y el Caribe (CILAC) celebrado en 2018, un espacio de debate e intercambio organizado por la Oficina Regional de Ciencias de la UNESCO promovió el diálogo entre altas autoridades de países latinoamericanos y caribeños y expertos en materia de políticas de datos abiertos de investigación y hardware científico abierto. La UNESCO, en colaboración con la Asociación Columbus, elaboró para esa ocasión un reporte sobre ciencia abierta para tomadores de decisión (Ramírez y Samoilovich, 2018). En el marco de CILAC 2018, en un evento paralelo al Foro Abierto se reunió un grupo de representantes de la academia y de organizaciones de la sociedad civil de diversos países de la región, quienes firmaron la "Declaración de Panamá sobre Ciencia Abierta". Esta declaración surge de manera contemporánea a la propuesta del "Plan S", iniciativa liderada por organizaciones financiadoras de la investigación europeas, cuyo objetivo es lograr que para 2021 la literatura publicada como resultados de investitigaciones financiadas con fondos públicos, esté disponible y visible para todos (OSI, 2019) y que no ha estado exenta de reacciones diversas en la región latinoamericana (Palma Peña, 2019). En este sentido, la "Declaración de Panamá" pone de manifiesto que "abrir la ciencia es ir más allá del acceso abierto" (Declaración de Panamá, 2018). Entre los postulados básicos se menciona la necesidad de transitar hacia modelos más colaborativos de creación, gestión, comunicación, preservación y apropiación entre el mundo académico, la sociedad y el Estado. En este contexto, los elementos que se definen como básicos para la implementación de la ciencia abierta, son: educación abierta; ciencia ciudadana; evaluación abierta; investigación abierta, reproducible y replicable; herramientas abiertas, políticas de software y hardware libres; infraestructuras abiertas; innovación de código abierto; licencias libres; acceso abierto; y datos abiertos de investiga- ción.

Al analizar los desarrollos en materia de ciencia abierta es posible pensar que, así como en cualquier otro campo del conocimiento, las políticas públicas implementadas son un reflejo del interés político y social por un tema determinado. Asimismo, los resultados científicos son también una muestra de la repercusión de determinada problemática en la comunidad académica. En este sentido, una de las metodologías más consolidadas para analizar la actividad investigadora es el estudio de las publicaciones científicas. En trabajos previos que analizan la evolución de las publicaciones sobre ciencia abierta en revistas indexadas en Web of Science y SCOPUS, se muestra que existe un interés muy reciente en estos temas. Asimismo, se evidencia que los documentos relacionados con acceso abierto son los más numerosos, aunque van ganando espacio otros temas como el de los datos abiertos (De Filippo y otros, 2019). Bajo el paraguas de las actividades realizadas en el marco de la ciencia abierta se han detectado también estudios bibliométricos centrados en el estudio de la producción sobre ciencia ciudadana (Follett y Strezov, 2015; Kullenberg y Kasperowski, 2016; Bautista-Puig y otros, 2019).

A partir del contexto planteado, los interrogantes que guían la investigación son:

- ¿Cuál es la situación que vive la región de Latinoamérica en cuanto a la implementación de políticas públicas sobre ciencia abierta?

- ¿Hay homogeneidad entre las políticas planteadas en cada país?

- ¿Se evidencia un interés por parte de la comunidad académica en temas de ciencia abierta que se haya plasmado en publicaciones científicas?

- ¿Cuáles son las principales temáticas que abordan estas publicaciones?

Para responderlos, en este trabajo se plantean dos objetivos generales:

- Identificar y analizar las principales políticas públicas sobre ciencia abierta en América Latina.

- Detectar la producción científica de la región sobre ciencia abierta y analizar sus principale características.

\section{Metodología}

Para alcanzar los objetivos generales se propone un análisis documental desde dos perspectivas. Por un lado, un estudio de la documentación proveniente de organismos oficiales que refleje las diferentes políticas, estrategias e iniciativas puestas en marcha en los países de Latinoamérica relacionadas con ciencia abierta y, por otro, el análisis de la producción 
científica que la comunidad académica está realizando sobre este tema. Ambos acercamientos resultan complementarios, no solo por el tipo de metodología y análisis utilizado (cualitativo en el primer caso y cuantitativo en el segundo), sino que permiten obtener un conocimiento más completo sobre la situación de la ciencia abierta en la región. Con estas dos perspectivas se intenta mostrar cómo se posicionan dos diferentes tipos de actores sociales (promotores de políticas públicas y académicos) en la implementación de la ciencia abierta en la región y cuáles son sus particularidades.

En el primer caso, para analizar el alcance del movimiento sobre ciencia abierta en Latinoamérica, se han recogido documentos oficiales producidos por los organismos que formulan y coordinan las principales políticas públicas implementadas y se han consultado páginas web de Ministerios y Agencias Nacionales y Federales de Ciencia y Tecnología y Consejos Nacionales de Investigación de la región. En función de tal recorte analítico, se han omitido explícitamente las iniciativas de políticas de ciencia abierta establecidas por organismos de coordinación de instituciones de educación superior, universidades u otro tipo de instituciones de los sistemas nacionales de ciencia y tecnología?.

Se ha revisado la situación en los 20 países que conforman la región latinoamericana: Argentina, Bolivia, Brasil, Chile, Colombia, Costa Rica, Cuba, Ecuador, El Salvador, Guatemala, Honduras, México, Nicaragua, Panamá, Paraguay, Puerto Rico, Perú, República Dominicana, Uruguay y Venezuela. La información recogida ha sido analizada a través de un estudio cualitativo comparativo y se ha estructurado en tablas y figuras que se organizan, tanto de manera cronológica, como por país. Se han identificado también las distintas iniciativas y se las ha clasificado en diferentes categorías de análisis en función de sus principales objetivos. En el apartado 3.1 se presentan, primero, los principales hitos en el desarrollo de acciones de Ciencia Abierta en los países de Latinoamérica, mencionando sus principales características y visualizando su evolución a través de una línea temporal (figura 1). A continuación, se presenta información de interés por país, indicando el tipo de iniciativa identificada, sus particularidades, el tipo de instituciones promotoras y el año de implementación (tabla 1). La información se muestra de manera cronológica (para poner en evidencia los avances a lo largo del tiempo).

En el segundo caso, para el análisis de la producción científica se ha usado como fuente de información la base de datos SCOPUS que incluye publicaciones de calidad y prestigio internacional. Esta fuente, además, ofrece una mayor cobertura documental de Latinoamérica que Web of Science y más flexibilidad a la hora de realizar búsquedas por palabras clave.

Para la recuperación documental se ha desarrollado una estrategia de búsqueda ad-hoc a partir de la identificación de términos clave recogidos en el título y las palabras clave de las publicaciones que ha sido consultada, probada y validada con expertos del área:

TITLE OR KEY = ( "open access" OR "open data" OR "citizen science" OR "open research" OR "open science" OR "citi-

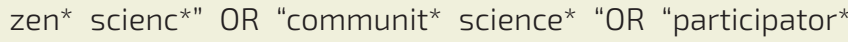
research*" OR "participator* action* research*" OR "communit* - based research*" OR "citizen* research*" OR "science* shop*" OR "citizen* scient*" OR "Public-participation" OR "open innovation" OR "open peer review" OR "open reproducibility" OR "open education resources" OR "open hardware for science")

Estas palabras clave fueron buscadas sobre la producción mundial y, a continuación, se han filtrado los documentos atendiendo al origen de la firma (se han seleccionado aquellos en los que existía al menos un firmante de un país latinoamericano) y la fecha (2000-2018). Se ha trabajado con todas las tipologías documentales e idiomas. Tras la depuración y normalización de datos se ha construido una base de datos relacional en MySQL conteniendo la información bibliográfica de cada uno de los documentos: título, autores, instituciones firmantes, revista de publicación, año, palabras clave, categoría temática, open access, etcétera. Una vez estructurada la información, a través de recuentos de frecuencias se han obtenido los siguientes indicadores bibliométricos que se presentan en el apartado 3.2.

- Producción por año y aportación de Latinoamérica al mundo: evolución anual de las publicaciones (figura 2).

- Número de documentos por país y año e índice de actividad (figura 3).

- Especialización temática de la región: distribución de las publicaciones sobre ciencia abierta en América Latina por categoría temática (figura 4).

- Principales palabras clave: frecuencia de aparición de clave de las publicaciones sobre ciencia abierta en América Latina (figura 5).

- Número y porcentaje de artículos open access por país y año (tabla 2).

\section{Resultados}

En este apartado se presentan los principales resultados obtenidos, tanto del estudio cualitativo exploratorio como del cuantitativo cienciométrico.

\subsection{Políticas públicas sobre Ciencia Abierta}

En este trabajo se entiende a las políticas públicas, siguiendo una concepción clásica en América Latina elaborada por 
Oscar Oszlak y Guillermo O'Donnell (1981), como un conjunto de estrategias y acciones a través de las cuales se expresan decisiones de una o más organizaciones estatales, simultáneas o sucesivas a lo largo del tiempo, que constituyen el modo de intervención del Estado frente a asuntos que concitan la atención, el interés o la movilización de otros actores de la sociedad civil. Las políticas públicas en materia de ciencia abierta, entonces, refieren a estrategias y acciones del Estado -específicamente, de sus organizaciones responsables de la formulación y coordinación de las políticas científicas y tecnológicas, de sus agencias financiadoras y de sus consejos nacionales de investigación- encaminadas a la promoción de sus principios y prácticas.

En diversas experiencias internacionales, inicialmente las políticas públicas sobre ciencia abierta estuvieron orientadas a la necesidad de difundir - de manera abierta- los resultados de investigación, partiendo del reconocimiento de que los hallazgos de la investigación financiada con recursos públicos deberían estar disponibles para el público sin restricción alguna. Actualmente, el alcance de las políticas de ciencia abierta se ha ampliado de tal forma que es posible encontrar políticas nacionales que impulsan una diversidad de prácticas de ciencia abierta, así como provisiones específicas en leyes, regulaciones o directrices (European Commission, 2017).

El análisis documental realizado a partir de la información proveniente de organismos oficiales, muestra que en Latinoamérica pueden identificarse diferentes iniciativas públicas en materia de políticas de ciencia abierta desde hace poco más de una década. Según material documental recogido de los Organismos Nacionales de Ciencia y Tecnología, sólo 6 de los 20 países son los más activos en este campo: se trata, en orden alfabético, de Argentina, Brasil, Chile, Colombia, México y Perú.

Buena parte de las acciones detectadas se vinculan con el desarrollo de Infraestructuras para acceso abierto a publicaciones, como en el caso de Brasil con el Portal Brasileño de Acceso Abierto a la Información Científica (OASISBR) cuya versión inicial se lanzó en 2007 (Leite, 2015) y la Biblioteca Digital Brasileña de Tesis y Disertaciones (BDTD) (IBICT, 2019). También es el caso del Repositorio Digital del Sistema de Información Científica de Chile, creado en 2010 y dirigido a capturar, preservar y difundir los resultados y la producción de los instrumentos financiados por la Comisión (CONICYT, 2019). En esta misma línea, un año más tarde en Argentina comienza a funcionar el Sistema Nacional de Repositorios Digitales (SNRD) cuyo propósito es conformar una red interoperable de repositorios digitales en ciencia y tecnología, a partir del establecimiento de políticas, estándares y protocolos comunes a todos los integrantes del SNRD (MECCYT, 2011).

También en 2011, Brasil es el primer país de la región en avanzar en la discusión de normativa para el acceso abierto. El Instituto Brasileño de Información en Ciencia y Tecnología (IBICT), centro de investigación del Ministerio de Ciencia, Tec- nología, Innovaciones y Comunicaciones (MCTIC) de Brasil, presenta al Parlamento un proyecto de ley que establece el proceso de registro y difusión de la producción técnico-científica por parte de instituciones de educación superior y de unidades de investigación (Senado Federal del Brasil, 2011). Sin embargo, esa normativa aún se encuentra en debate.

En 2012, Colombia pone en marcha la Estrategia Nacional de Recursos Educativos Digitales Abiertos (REDA), una iniciativa única en la región, dirigida a promover el desarrollo de capacidades para la producción y gestión de recursos educativos digitales bajo un licenciamiento de acceso abierto que permite y promueve su uso, adaptación, modificación y/o personalización (MinEducación, 2013).

En materia de normativa para el acceso abierto, en 2013 en Perú se promulga la Ley que regula el Repositorio Nacional Digital de Ciencia, Tecnología e Innovación de Acceso Abierto, estableciendo la obligación de publicar los resultados de todas las investigaciones científicas financiadas por fuentes estatales en el repositorio digital nacional, el cual es interoperable con otros repositorios regionales y globales (Congreso de la República de Perú, 2013), reglamentada dos años más tarde (Presidencia del Consejo de Ministros de Perú, 2015). Unos meses más tarde que Perú, en 2013 en Argentina se sanciona la Ley 26.899 de Creación de Repositorios Digitales Institucionales de Acceso Abierto, Propios o Compartidos, que obliga a las instituciones que reciben financiamiento del Estado Nacional a crear repositorios digitales institucionales de acceso abierto en los que se deposite la producción científico-tecnológica y los datos primarios de las investigaciones después de cinco años de su recopilación, para que otros investigadores puedan usarlos (Honorable Congreso de la Nación Argentina, 2013).

En 2014 Chile lanza la Propuesta de Política de Datos Abiertos y las recomendaciones para el acceso y preservación de información científica y datos de investigación (CONICYT, 2014). 2014 es también un año clave para México ya que se concreta la Modificación de la Ley de Ciencia y Tecnología, la Ley General de Educación y la Ley Orgánica del Consejo Nacional de Ciencia y Tecnología (CONACYT) para promover el acceso abierto a todo el conocimiento generado con fondos públicos (México, 2014). Con ella, se amplían las facultades del CONACYT con el fin de desarrollar una estrategia nacional para la democratización de la información científica y para establecer criterios de calidad y estándares técnicos para la creación de repositorios digitales. También se establecen las bases para la creación de un repositorio nacional de acceso abierto operado por CONACYT (CONACYT, 2014), que es lanzado recién en 2019.

En 2015, Colombia pone en marcha el Sistema Nacional de Acceso Abierto al Conocimiento (SNAAC) con el objetivo de fomentar y fortalecer las condiciones y capacidades para la producción, articulación, organización, visibilidad, promoción del uso y la visibilidad de la producción científica colombiana 
en acceso abierto (MinEducación, 2019).

En 2016 en Perú comienza a funcionar la Red Nacional de Repositorios Digitales de Ciencia, Tecnología e Innovación de Acceso Abierto (RENARE) y el Repositorio Nacional Digital de Ciencia, Tecnología e Innovación de Acceso Abierto (ALICIA, Acceso Libre a Información Científica para la Innovación) con el fin de conformar una red nacional interoperable de repositorios digitales, a partir del establecimiento de políticas, estándares y protocolos comunes a todos los integrantes de la Red (CONCYTEC, 2017, 2019). Es también en 2016 que Argentina reglamenta la Ley 26.899 de acceso abierto, estableciendo plazos y pautas del funcionamiento de los repositorios digitales para que los actores alcanzados den cumplimiento a la normativa (MECCYT, 2016) y en Brasil el IBICT logra avanzar en la formulación de un Manifiesto de acceso abierto a datos de investigación brasileños para ciencia ciudadana, adoptando una posición favorable al acceso abierto a la información y datos. Un año más tarde, en 2017, en Argentina se pone en marcha el Programa de Datos Abiertos en Ciencia y Tecnología con el propósito de promover, formular y ejecutar la estrategia nacional de ciencia abierta y ciudadana del país (MECCYT, 2017a) y se lanza la versión beta del Portal de Información de Ciencia y Tecnología Argentino, orientada a distintos tipos de usuarios de visualizaciones y datos abiertos en ciencia y tecnología (MECCYT, 2017b). Asimismo, se introduce la obligatoriedad gradual de presentación de un Plan de Gestión de Datos recolectados o producidos en el curso de un proyecto de investigación a ser financiado por el Consejo Nacional de
Investigaciones Científicas y Técnicas (CONICET), iniciativa pionera en Argentina implementada a través de una prueba piloto aplicada a Proyectos de Unidades Ejecutoras (Actis y Carlino, 2017).

Simultáneamente, en Brasil se introduce también la obligatoriedad gradual de presentación de un Plan de Gestión de Datos en las solicitudes de financiamiento remitidas a la Fundación de Apoyo a la Investigación Científica del Estado de São Paulo (FAPESP) (FAPESP, 2017). Se trata de una iniciativa pionera en Brasil implementada a través de una convocatoria aplicada a Proyectos Temáticos, extendida posteriormente a otras modalidades de ayuda a proyectos y becas doctorales y posdoctorales de acuerdo con la Política de acceso abierto a las publicaciones resultantes de los subsidios y becas de investigación FAPESP instituida en 2019 (FAPESP, 2019). En México también se presentan los Lineamientos Generales de Ciencia Abierta y los Lineamientos Jurídicos de Ciencia Abierta del CONACYT con el propósito de coordinar las políticas, los recursos, los programas y las acciones realizadas por el Consejo relacionadas con la ciencia abierta (CONACYT, 2017a, 2017b).

En 2018 en Argentina el Programa de Datos Abiertos en Ciencia y Tecnología institucionalizó un novedoso Ciclo de Charlas y Talleres sobre Ciencia Abierta y Ciudadana con una agenda variada de temas, organizado regularmente en colaboración con el Centro de Investigaciones para la Transformación (CENIT) y Cientópolis (MECCYT, 2019) y el Instituto Antártico Argentino (IAA) institucionaliza su Plan de Gestión de Datos sobre la base de su Planilla de Relevamiento Previo a Campaña

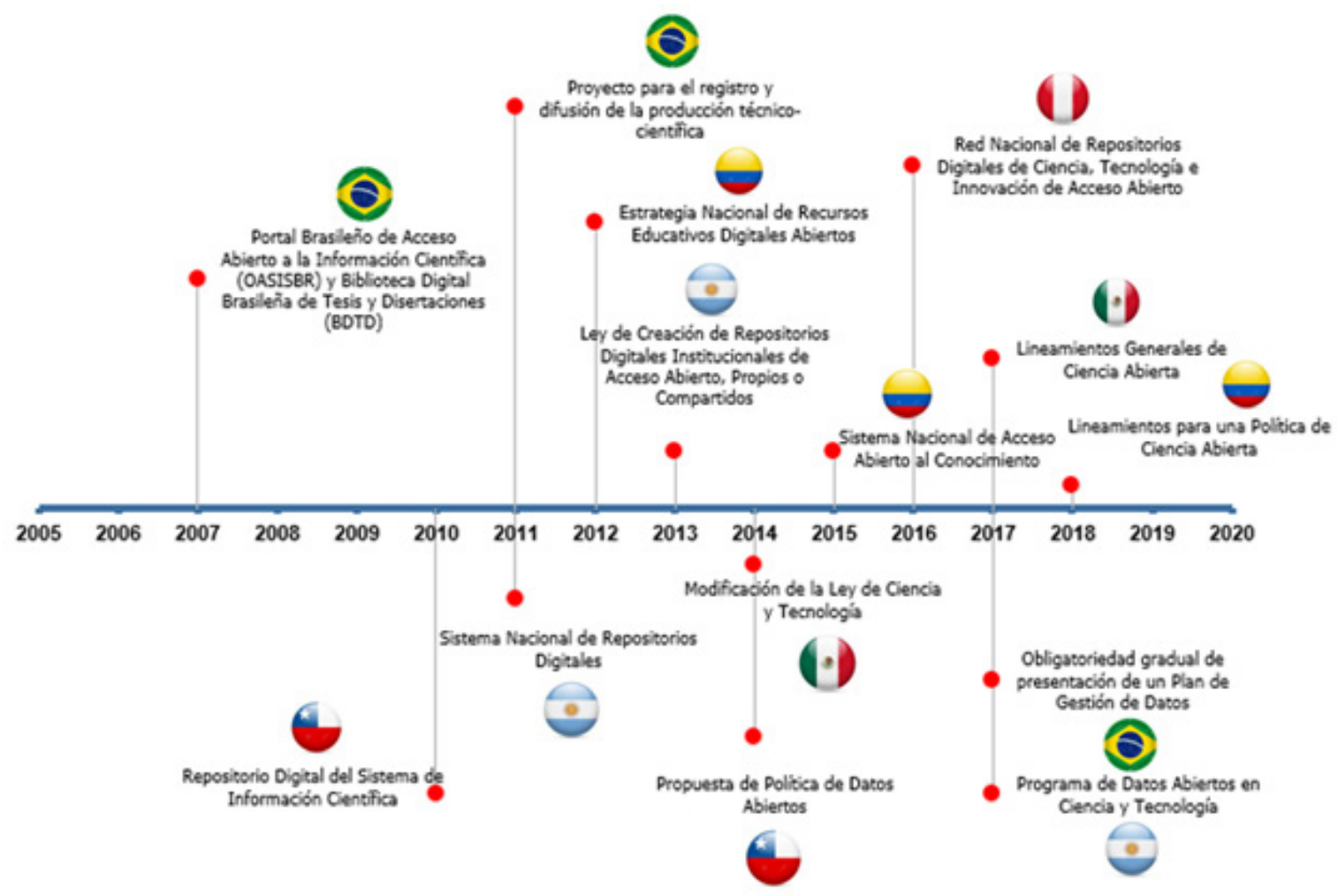

Figura 1. Principales hitos en el desarrollo de acciones de Ciencia Abierta en los países de Latinoamérica. Elaboración propia. 
y Declaración de Datos Resultantes de la Ejecución de Proyectos Científicos en la Antártida (IAA, 2018). En Colombia ese año se trazan los lineamientos para una Política de Ciencia Abierta identificando una serie de aspectos en los que se debe trabajar para generar condiciones habilitantes para el desarrollo de la ciencia abierta en el país y se hacen algunas propuestas iniciales para un plan de trabajo más detallado con la comunidad de interés proyectado a 2030 (COLCIENCIAS, 2018). En Uruguay ese mismo año y desde la Agencia Nacional de Investigación e Innovación se formula el Proyecto de Sistema Nacional de Repositorios Digitales de Información Científico-Tecnoló- gica, que avanza sobre el diseño de un plan para desarrollar e implementar un sistema nacional de repositorios digitales que articule las acciones tendientes a la definición de políticas, el desarrollo de infraestructura, la coordinación de acciones y la adopción de planes, programas y proyectos relacionados con el acceso abierto a la producción científica nacional (Fernández, 2019).

En la figura 1 se muestra la cronología de los principales hitos en materia de Ciencia Abierta en la región y en la Tabla 1 se ofrece información complementaria sobre cada una de las iniciativas mencionadas.

\begin{tabular}{|c|c|c|c|c|}
\hline País & Tipo de iniciativa & Descripción de la iniciativa & $\begin{array}{c}\text { Tipos de instituciones } \\
\text { promotoras }\end{array}$ & Año \\
\hline \multirow[t]{5}{*}{ Argentina } & $\begin{array}{l}\text { Infraestructura } \\
\text { para acceso abierto } \\
\text { a publicaciones }\end{array}$ & $\begin{array}{l}\text { Sistema Nacional de Repositorios Digita- } \\
\text { les de la Secretaría de Gobierno de Ciencia, } \\
\text { Tecnología e Innovación Productiva (SGCTIP) } \\
\text { del Ministerio de Educación, Cultura, Ciencia } \\
\text { y Tecnología conjuntamente con el Consejo } \\
\text { Interinstitucional de Ciencia y Tecnología }\end{array}$ & $\begin{array}{l}\text { Entidad gubernamental de } \\
\text { política de ciencia y tecnología }\end{array}$ & 2011 \\
\hline & $\begin{array}{l}\text { Normativa para acceso } \\
\text { abierto a publicaciones } \\
\text { y datos de investigación }\end{array}$ & $\begin{array}{l}\text { Ley 26,899 de Creación de Repositorios } \\
\text { Digitales Institucionales de Acceso Abierto, } \\
\text { Propios o Compartidos, reglamentada por } \\
\text { Resolución } 753 \text { - E/2016 del MECCYT de } 2016\end{array}$ & $\begin{array}{l}\text { Parlamento y entidad guber- } \\
\text { namental de política de } \\
\text { ciencia y tecnología }\end{array}$ & 2013 \\
\hline & $\begin{array}{l}\text { Articulación de polí- } \\
\text { ticas de promoción } \\
\text { de ciencia abierta }\end{array}$ & $\begin{array}{l}\text { Programa de Datos Abiertos en } \\
\text { Ciencia y Tecnología creado por Reso- } \\
\text { lución 640/2017 de la SGCTIP }\end{array}$ & $\begin{array}{l}\text { Entidad gubernamental de } \\
\text { política de ciencia y tecnología }\end{array}$ & 2017 \\
\hline & \multirow[t]{2}{*}{$\begin{array}{l}\text { Planificación de } \\
\text { apertura de datos } \\
\text { de investigación en } \\
\text { convocatoria de finan- } \\
\text { ciamiento de proyectos }\end{array}$} & $\begin{array}{l}\text { Obligatoriedad gradual de presentación } \\
\text { de un Plan de Gestión de Datos reco- } \\
\text { lectados o producidos en el curso de un } \\
\text { proyecto de investigación a ser financiado } \\
\text { por el Consejo Nacional de Investigacio- } \\
\text { nes Científicas y Técnicas (CONICET) }\end{array}$ & $\begin{array}{l}\text { Entidad gubernamental de } \\
\text { financiamiento y ejecución } \\
\text { de ciencia y tecnología }\end{array}$ & 2017 \\
\hline & & $\begin{array}{l}\text { Obligatoriedad de presentación de un Plan } \\
\text { de Gestión de Datos producidos en el curso } \\
\text { de campañas y proyectos científicos en la } \\
\text { Antártida por personal del Instituto Antártico } \\
\text { Argentino (IAA) y Política del Repositorio } \\
\text { Digital Institucional del IAA de Acceso Abierto } \\
\text { a las Publicaciones y Datos Primarios }\end{array}$ & $\begin{array}{l}\text { Entidad gubernamental de } \\
\text { ejecución de ciencia y tecnología }\end{array}$ & 2018 \\
\hline Brasil & $\begin{array}{l}\text { Infraestructura } \\
\text { para acceso abierto } \\
\text { a publicaciones }\end{array}$ & $\begin{array}{l}\text { Portal Brasileño de Acceso Abierto a la } \\
\text { Información Científica y Biblioteca Digital } \\
\text { Brasileña de Tesis y DIsertaciones del Insti- } \\
\text { tuto Brasileño de Información en Ciencia y } \\
\text { Tecnología (IBICT) del Ministerio de Ciencia, } \\
\text { Tecnología, Innovaciones y Comunicaciones } \\
\text { (MCTIC). Apoyo a Repositorios Institucio- } \\
\text { nales Brasileños y a la conformación del } \\
\text { Repositorio Común de Brasil (DEPOSITA) } \\
\text { para los investigadores que no tienen sus } \\
\text { propios repositorios institucionales }\end{array}$ & $\begin{array}{l}\text { Instituto gubernamen- } \\
\text { tal de investigación }\end{array}$ & 2007 \\
\hline
\end{tabular}




\begin{tabular}{|c|c|c|c|c|}
\hline & $\begin{array}{l}\text { Proyecto de normativa } \\
\text { de acceso abierto } \\
\text { a publicaciones }\end{array}$ & $\begin{array}{l}\text { Proyecto promovido por el IBICT del } \\
\text { MCTIC, en debate. Establece el proceso } \\
\text { de registro y difusión de la producción } \\
\text { técnico-científica por parte de } \\
\text { instituciones de educación superior y de } \\
\text { unidades de investigación en Brasil }\end{array}$ & $\begin{array}{l}\text { Parlamento e instituto } \\
\text { gubernamental de investigación }\end{array}$ & 2011 \\
\hline & $\begin{array}{l}\text { Planificación de apertura } \\
\text { de datos de investiga- } \\
\text { ción en convocatoria } \\
\text { de financiamiento de } \\
\text { proyectos y becas }\end{array}$ & $\begin{array}{l}\text { Obligatoriedad gradual de presentación } \\
\text { de un Plan de Gestión de Datos en las } \\
\text { solicitudes de financiamiento remitidas a } \\
\text { la Fundación de Apoyo a la Investigación } \\
\text { Científica del Estado de São Paulo (FAPESP) }\end{array}$ & $\begin{array}{l}\text { Agencia gubernamental } \\
\text { de financiamiento de } \\
\text { ciencia y tecnología }\end{array}$ & 2017 \\
\hline \multirow[t]{2}{*}{ Chile } & $\begin{array}{l}\text { Infraestructura } \\
\text { para acceso abierto } \\
\text { a publicaciones }\end{array}$ & $\begin{array}{l}\text { Repositorio Digital del Sistema de } \\
\text { Información Científica (SIC) de la } \\
\text { Comisión Nacional de Investigación } \\
\text { Científica y Tecnológica (CONICYT) }\end{array}$ & $\begin{array}{l}\text { Entidad gubernamental de } \\
\text { política de ciencia y tecnología }\end{array}$ & 2010 \\
\hline & $\begin{array}{l}\text { Promoción de políticas } \\
\text { de apertura de datos } \\
\text { de investigación }\end{array}$ & $\begin{array}{l}\text { Propuesta de Política de Datos Abiertos } \\
\text { del Programa de Información Científica } \\
\text { de la CONICYT y recomendaciones para } \\
\text { el acceso y preservación de información } \\
\text { científica y datos de investigación } \\
\text { mediante el Manual de Datos Abiertos }\end{array}$ & $\begin{array}{l}\text { Entidad gubernamental de } \\
\text { política de ciencia y tecnología }\end{array}$ & 2014 \\
\hline \multirow[t]{3}{*}{ Colombia } & $\begin{array}{l}\text { Promoción de recursos } \\
\text { educacionales abiertos }\end{array}$ & $\begin{array}{l}\text { Estrategia Nacional de Recursos Educativos } \\
\text { Digitales Abiertos (REDA) del Ministerio } \\
\text { de Educación con la colaboración de la } \\
\text { Corporación Red Nacional Académica } \\
\text { de Tecnología Avanzada (RENATA) }\end{array}$ & $\begin{array}{l}\text { Entidad gubernamental de } \\
\text { política de educación superior }\end{array}$ & 2012 \\
\hline & $\begin{array}{l}\text { Infraestructura } \\
\text { para acceso abierto } \\
\text { a publicaciones }\end{array}$ & $\begin{array}{l}\text { Sistema Nacional de Acceso Abierto al } \\
\text { Conocimiento del Ministerio de Educación y } \\
\text { el Departamento Administrativo de Ciencia, } \\
\text { Tecnología e Innovación (COLCIENCIAS) } \\
\text { con la colaboración de RENATA }\end{array}$ & $\begin{array}{l}\text { Entidades gubernamentales } \\
\text { de política de educación supe- } \\
\text { rior y de ciencia y tecnología }\end{array}$ & 2015 \\
\hline & $\begin{array}{l}\text { Articulación de polí- } \\
\text { ticas de promoción } \\
\text { de ciencia abierta }\end{array}$ & $\begin{array}{l}\text { Lineamientos para una Política de Ciencia } \\
\text { Abierta en Colombia de COLCIENCIAS }\end{array}$ & $\begin{array}{l}\text { Entidad gubernamental de } \\
\text { política de ciencia y tecnología }\end{array}$ & 2018 \\
\hline \multirow[t]{3}{*}{ México } & $\begin{array}{l}\text { Normativa de acceso } \\
\text { abierto a publicaciones }\end{array}$ & $\begin{array}{l}\text { Modificación de la Ley de Ciencia y } \\
\text { Tecnología, la Ley General de Educación } \\
\text { y la Ley Orgánica del Consejo Nacional } \\
\text { de Ciencia y Tecnología (CONACYT) }\end{array}$ & Parlamento & 2014 \\
\hline & $\begin{array}{l}\text { Infraestructura } \\
\text { para acceso abierto } \\
\text { a publicaciones }\end{array}$ & $\begin{array}{l}\text { Lineamientos Generales para el Repositorio } \\
\text { Nacional y los Repositorios Institucionales } \\
\text { del CONACYT en } 2014 \text { y lanzamiento } \\
\text { del Repositorio Nacional en } 2019\end{array}$ & $\begin{array}{l}\text { Entidad gubernamental de } \\
\text { política de ciencia y tecnología }\end{array}$ & 2014 \\
\hline & $\begin{array}{l}\text { Articulación de polí- } \\
\text { ticas de promoción } \\
\text { de ciencia abierta }\end{array}$ & $\begin{array}{l}\text { Lineamientos Generales de Ciencia Abierta } \\
\text { y Lineamientos Jurídicos de Ciencia } \\
\text { Abierta del CONACYT con el propósito de } \\
\text { coordinar las políticas, recursos, programas } \\
\text { y acciones realizadas por el Consejo } \\
\text { relacionadas con la ciencia abierta }\end{array}$ & $\begin{array}{l}\text { Entidad gubernamental de } \\
\text { política de ciencia y tecnología }\end{array}$ & 2017 \\
\hline Perú & $\begin{array}{l}\text { Normativa de acceso } \\
\text { abierto a publicaciones }\end{array}$ & $\begin{array}{l}\text { Ley que regula el Repositorio Nacional } \\
\text { Digital de Ciencia, Tecnología e Innovación de } \\
\text { Acceso Abierto, reglamentada por Decreto } \\
\text { Supremo 006-2015 de la Presidencia }\end{array}$ & $\begin{array}{l}\text { Parlamento y presidencia } \\
\text { del poder ejecutivo }\end{array}$ & 2013 \\
\hline
\end{tabular}




\begin{tabular}{|l|l|l|l|}
\hline & $\begin{array}{l}\text { Infraestructura } \\
\text { para acceso abierto } \\
\text { a publicaciones }\end{array}$ & $\begin{array}{l}\text { Red Nacional de Repositorios Digitales de } \\
\text { Ciencia, Tecnología e Innovación de Acceso } \\
\text { Abierto (RENARE) y Repositorio Nacional } \\
\text { Digital de Ciencia, Tecnología e Innovación } \\
\text { de Acceso Abierto (ALICIA, Acceso Libre a } \\
\text { Información Científica para la Innovación) } \\
\text { del Consejo Nacional de Ciencia, Tecnolo- } \\
\text { gía e Innovación Tecnológica (CONCYTEC) }\end{array}$ & $\begin{array}{l}\text { Entidad gubernamental de } \\
\text { política de ciencia y tecnología }\end{array}$ \\
\hline Uruguay & $\begin{array}{l}\text { Proyecto de infraestruc- } \\
\text { tura para acceso abierto }\end{array}$ & $\begin{array}{l}\text { Proyecto de Sistema Nacional de Repo- } \\
\text { sitorios Digitales de Información } \\
\text { Científico-Tecnológica de la Agencia Nacio- } \\
\text { nal de Investigación e Innovación (ANNI) }\end{array}$ & $\begin{array}{l}\text { Agencia gubernamen- } \\
\text { tal de financiamiento de } \\
\text { ciencia y tecnología }\end{array}$ \\
\hline
\end{tabular}

Tabla 1. Principales iniciativas de política pública sobre ciencia abierta en América Latina por país. Elaboración propia.

\subsection{Publicaciones sobre ciencia abierta}

En cuanto al análisis de las publicaciones científicas recogidas en SCOPUS, entre 2000 y 2018 se detectaron 1334 documentos de Latinoamérica sobre ciencia abierta (un $4 \%$ de la producción mundial). Más de dos tercios de esta producción se concentra en los últimos 5 años y el aporte de la región al mundo ha ido en constante crecimiento (en torno al 2\%-3\% hasta 2014 y cercana al 6\% en 2018) (Ver figura 2).

La distribución de la producción por países muestra que, aunque se han detectado publicaciones sobre Ciencia Abierta en todos los países latinoamericanos, Brasil es el primer productor de la región (puesto 15 del mundo) con 576 documentos (un 43\% de LAT). Le siguen México (214), Colombia (131), Chile (111) y Argentina (89).

Para relativizar el volumen de producción en función del tamaño de los países, se ha usado el Índice de Actividad (IA). Este indicador permite comparar la proporción de documentos de cada país sobre un tema (en este caso ciencia abierta) frente a la proporción de publicaciones totales de cada país en SCOPUS. Se aprecia que, entre los países con más de 5 documentos en el período de análisis, los más intensivos en la producción sobre ciencia abierta son: Ecuador, Bolivia, Guatemala, Perú, Costa Rica y Paraguay, con Índices de Actividad superiores a 1. En la Figura 3 se muestra la distribución de las publicaciones por país y se indican los valores de IA más elevados.

En cuanto a la especialización temática, medida en términos de categorías de las revistas de publicación, se observa que Computer Science y Social Sciences son las áreas en las que se concentra la producción con poco más de un 30\% de los documentos cada una. Aunque las tres primeras áreas de especialización de Latinoamérica son las mismas que en el total de la producción mundial sobre ciencia abierta, las proporciones varían tal como se puede apreciar en la figura 4.

En las publicaciones se han detectado 5650 palabras clave de autor, tras su normalización y estandarización se han realizado análisis de frecuencia. Los términos más frecuentes han

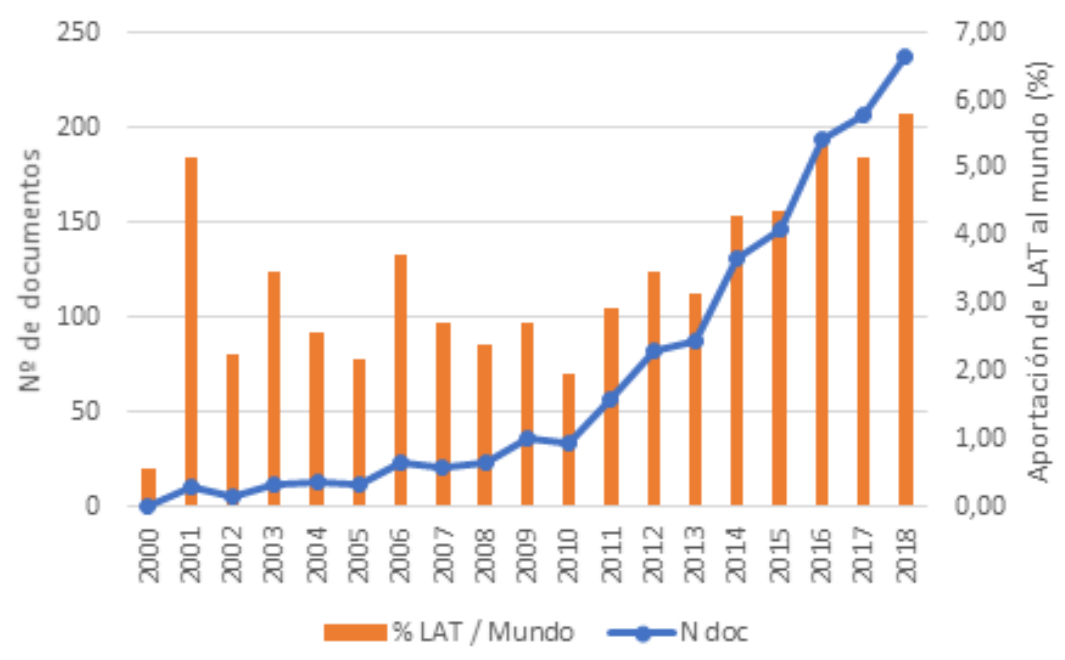

Figura 2. Evolución de las publicaciones sobre ciencia abierta en América Latina, 2000-2018. Elaboración propia. 


\begin{tabular}{|l|c|c|c|c|}
\hline \multicolumn{1}{|c|}{ PAIS } & N doc & $\begin{array}{c}\text { \% de } \\
\text { LAT }\end{array}$ & $\begin{array}{c}\% \\
\text { mundo }\end{array}$ & IA \\
\hline Brasil & 576 & 43,15 & 1,77 & 0,87 \\
\hline México & 214 & 16,03 & 0,66 & 0,98 \\
\hline Colombia & 131 & 9,81 & 0,40 & $\mathbf{1 , 8 3}$ \\
\hline Chile & 111 & 8,31 & 0,34 & 1,06 \\
\hline Argentina & 89 & 6,67 & 0,27 & 0,63 \\
\hline Ecuador & 68 & 5,09 & 0,21 & $\mathbf{5 , 0 7}$ \\
\hline Perú & 56 & 4,19 & 0,17 & $\mathbf{3 , 1 7}$ \\
\hline Cuba & 32 & 2,40 & 0,10 & $\mathbf{1 , 2 1}$ \\
\hline Costa Rica & 27 & 2,02 & 0,08 & $\mathbf{3 , 0 6}$ \\
\hline Venezuela & 23 & 1,72 & 0,07 & 0,87 \\
\hline Puerto Rico & 17 & 1,27 & 0,05 & 1,52 \\
\hline Bolivia & 15 & 1,12 & 0,05 & $\mathbf{4 , 6 0}$ \\
\hline Uruguay & 14 & 1,05 & 0,04 & 1,04 \\
\hline Jamaica & 10 & 0,75 & 0,03 & $\mathbf{2 , 2 8}$ \\
\hline Guatemala & 8 & 0,60 & 0,02 & $\mathbf{3 , 5 1}$ \\
\hline Trinidad y Tobago & 8 & 0,60 & 0,02 & $\mathbf{1 , 7 3}$ \\
\hline Honduras & 7 & 0,52 & 0,02 & \\
\hline Paraguay & 5 & 0,37 & 0,02 & $\mathbf{2 , 9 4}$ \\
\hline Barbados & 4 & 0,30 & 0,01 & \\
\hline El Salvador & 4 & 0,30 & 0,01 & $\mathbf{3 , 4 5}$ \\
\hline Haití & 4 & 0,30 & 0,01 & \\
\hline Nicaragua & 4 & 0,30 & 0,01 & $\mathbf{3 , 2 9}$ \\
\hline Bahamas & 2 & 0,15 & 0,01 & \\
\hline Belice & 2 & 0,15 & 0,01 & \\
\hline Panamá & 1 & 0,07 & 0,00 & 0,20 \\
\hline
\end{tabular}

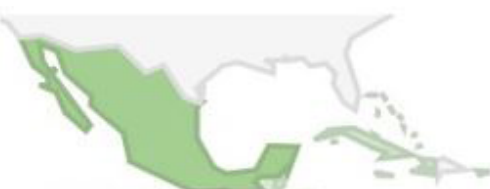

Guatemala $I A=3,5$
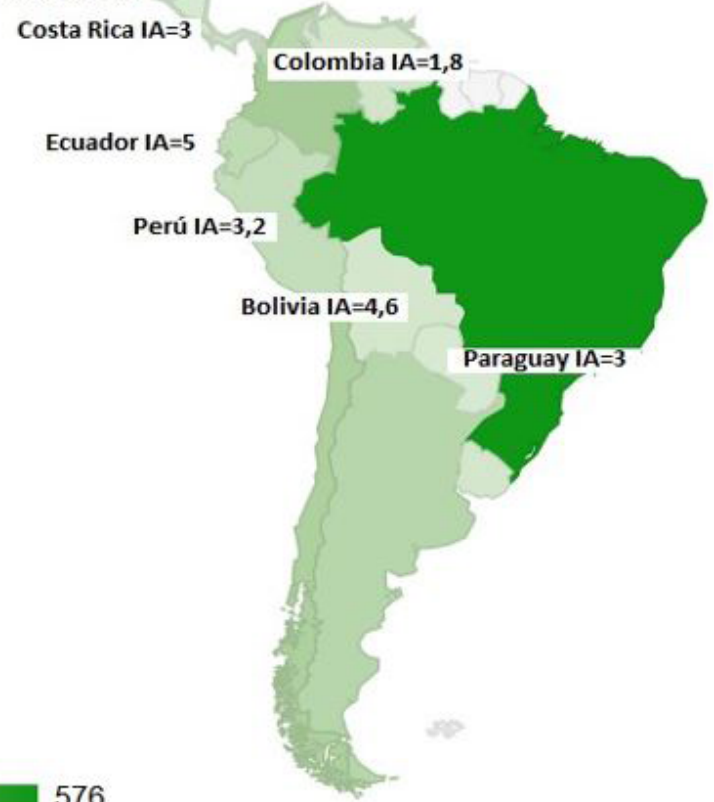

Figura 3. Distribución de las publicaciones sobre ciencia abierta en América Latina por país. Elaboración propia.

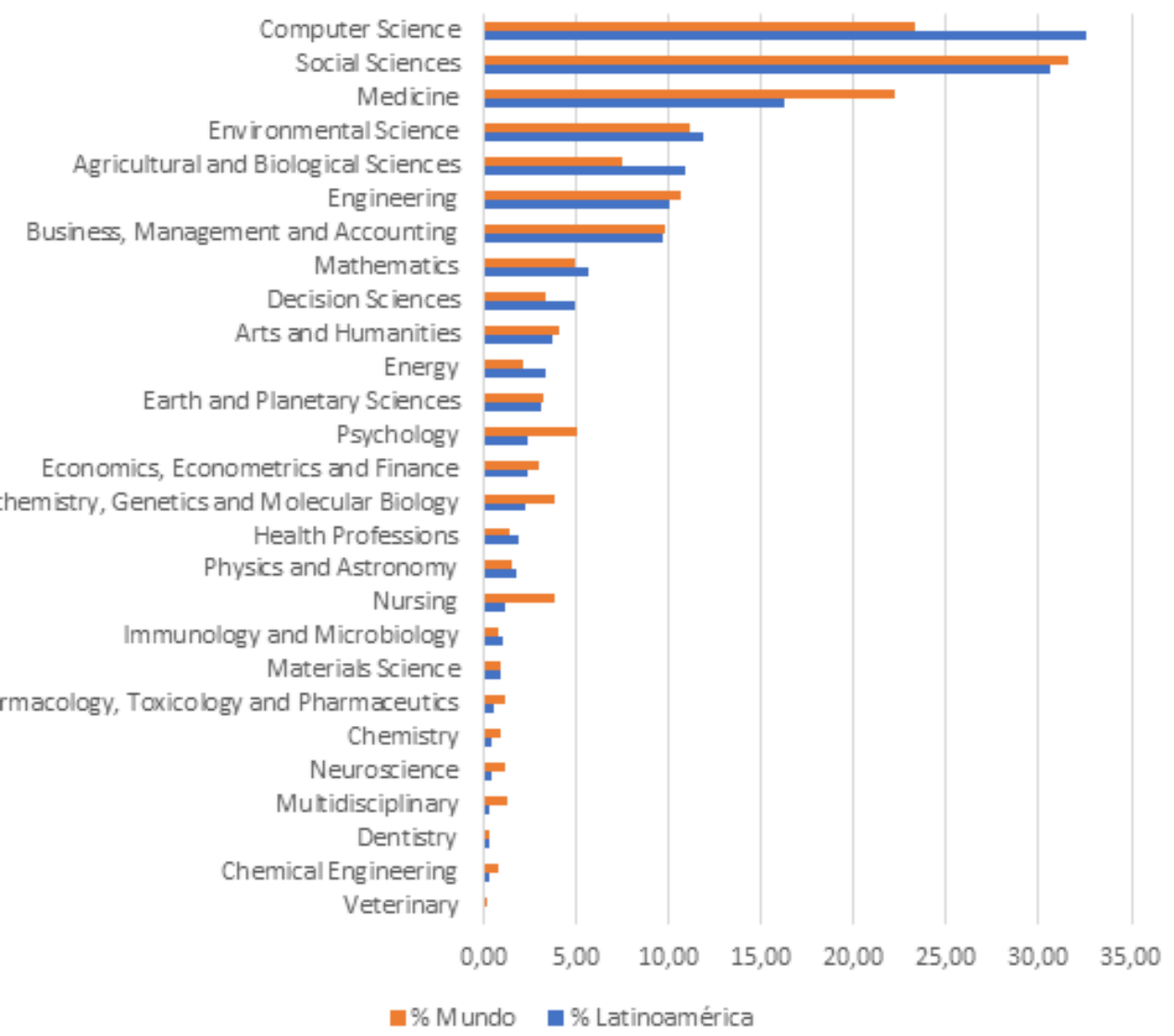

Figura 4. Distribución de las publicaciones sobre ciencia abierta en América Latina por categoría temática. Elaboración propia. 


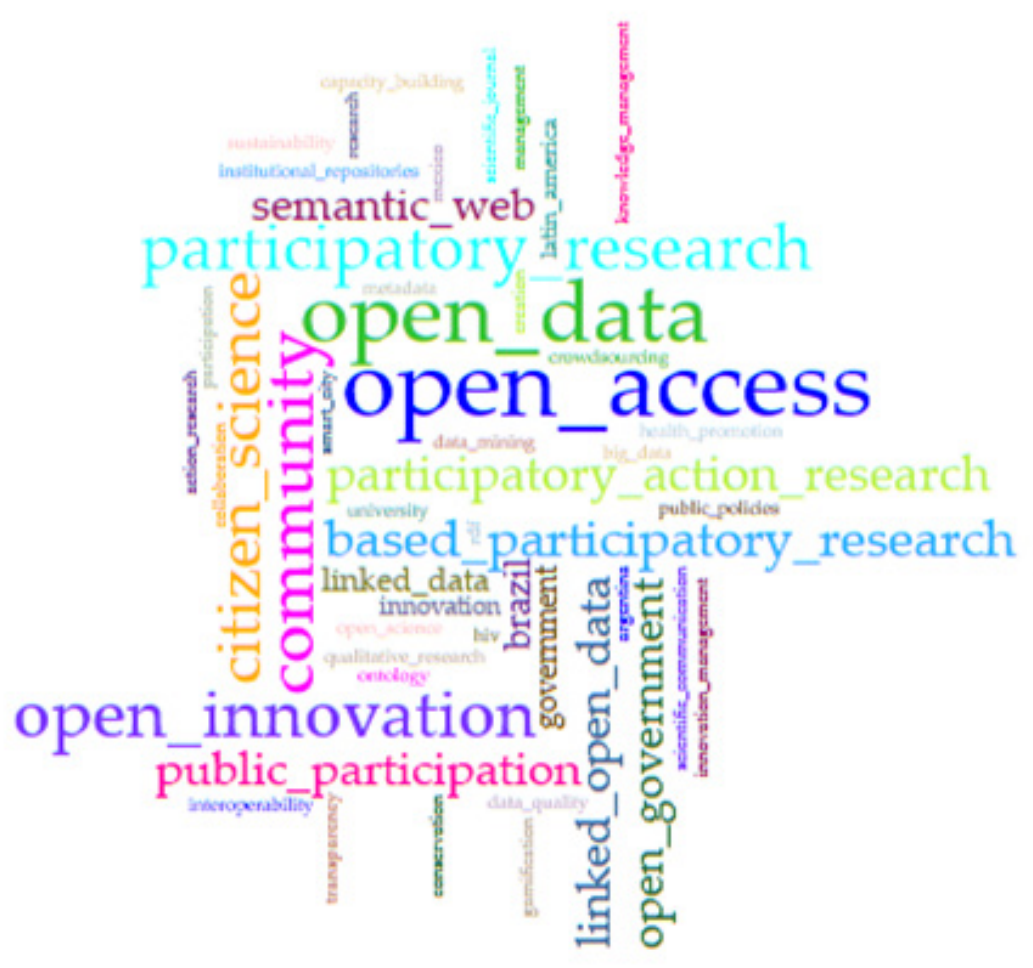

\begin{tabular}{|l|l|}
\hline Términos & Contar \\
\hline open_access & 162 \\
\hline open_data & 140 \\
\hline open_innovation & 99 \\
\hline participatory_research & 99 \\
\hline community & 95 \\
\hline citizen_science & 86 \\
\hline based_participatory_research & 67 \\
\hline participatory_action_research & 64 \\
\hline public_participation & 60 \\
\hline linked_open_data & 55 \\
\hline open_government & 52 \\
\hline semantic_web & 50 \\
\hline brazil & 44 \\
\hline linked_data & 36 \\
\hline government & 33 \\
\hline innovation & 29 \\
\hline latin_america & 27 \\
\hline transparency & 23 \\
\hline mexico & 21 \\
\hline rdf & 20 \\
\hline participation & 18 \\
\hline open_science & 17 \\
\hline big_data & 16 \\
\hline qualitative_research & \\
\hline & \\
\hline
\end{tabular}

Figura 5. Frecuencia de aparición de palabras clave en publicaciones sobre ciencia abierta en América Latina. Elaboración propia.

sido: Open Access (162 documentos), Open Data (140), Open Innovation (99) y Participatory research (99) (Figura 5).

Si se consideran aspectos relacionados con el acceso abierto a los documentos, se observa que Latinoamérica presenta valores de open access superiores al resto del mundo. Entre los años 2000 y 2018 un 25,7\% de las publicaciones de la región fueron $\mathrm{OA}$ frente al 15,3\% en el total mundial. Estas cifras varían considerablemente al analizar las publicaciones sobre ciencia abierta. Si bien cabría esperar que la producción sobre este tema se difundiera en abierto, en Latinoamérica el OA alcanza el 23,9 (casi 2 puntos porcentuales por debajo de la producción total de la región en SCOPUS). Por el contrario, en el resto del mundo los valores se incrementan en más de 4 puntos porcentuales con respecto a las publicaciones totales en la base de datos (Tabla 2).

\begin{tabular}{|l|l|l|}
\hline \multicolumn{1}{|c|}{ Tipo de Acceso } & \multicolumn{1}{|c|}{ Total Docs. LAT } & No docs. LAT OS. \\
\hline Open Access & 427.815 & 319 \\
\hline NO Open Access & 1234087 & 1016 \\
\hline \%OA & $25,74 \%$ & $23,90 \%$ \\
\hline TOTAL & 1.661 .902 & 1.335 \\
\hline
\end{tabular}

Tabla 2. Publicaciones de Latinoamérica en Open Access. Elaboración propia.
La distribución de las publicaciones en acceso abierto por países muestra que, entre los que publican más de 5 documentos sobre ciencia abierta, destacan las proporciones de Open Access en Guatemala y Honduras. Entre los grandes productores, el porcentaje de publicaciones en Open Access no alcanza el 30\% (figura 6).

\section{Discusión}

Sin dudas el interés por el movimiento de ciencia abierta está teniendo un impacto notable en todo el mundo y Latinoamérica no es la excepción. Para analizar en mayor profundidad cuál es la situación de la región, en este estudio se ha adoptado un acercamiento basado en dos perspectivas complementarias que intentan detectar cuál es el grado de desarrollo y la orientación de las políticas públicas sobre ciencia abierta, y cómo se posiciona la región en cuanto a su producción científica sobre el tema.

Al analizar bibliografía sobre ciencia abierta en Latinoamérica, es posible advertir que, entre las diferentes iniciativas existentes, autores e instituciones de la región destacan como elementos clave el acceso abierto, los datos abiertos, la investigación participativa y la ciencia ciudadana (STEPS, 2016). Sin embargo, aunque también se mencionan otros factores de importancia como la necesidad de contar con métricas alternativas para la evaluación de la investigación, los recursos educativos en abierto, y la ética y la transparencia en el pro- 


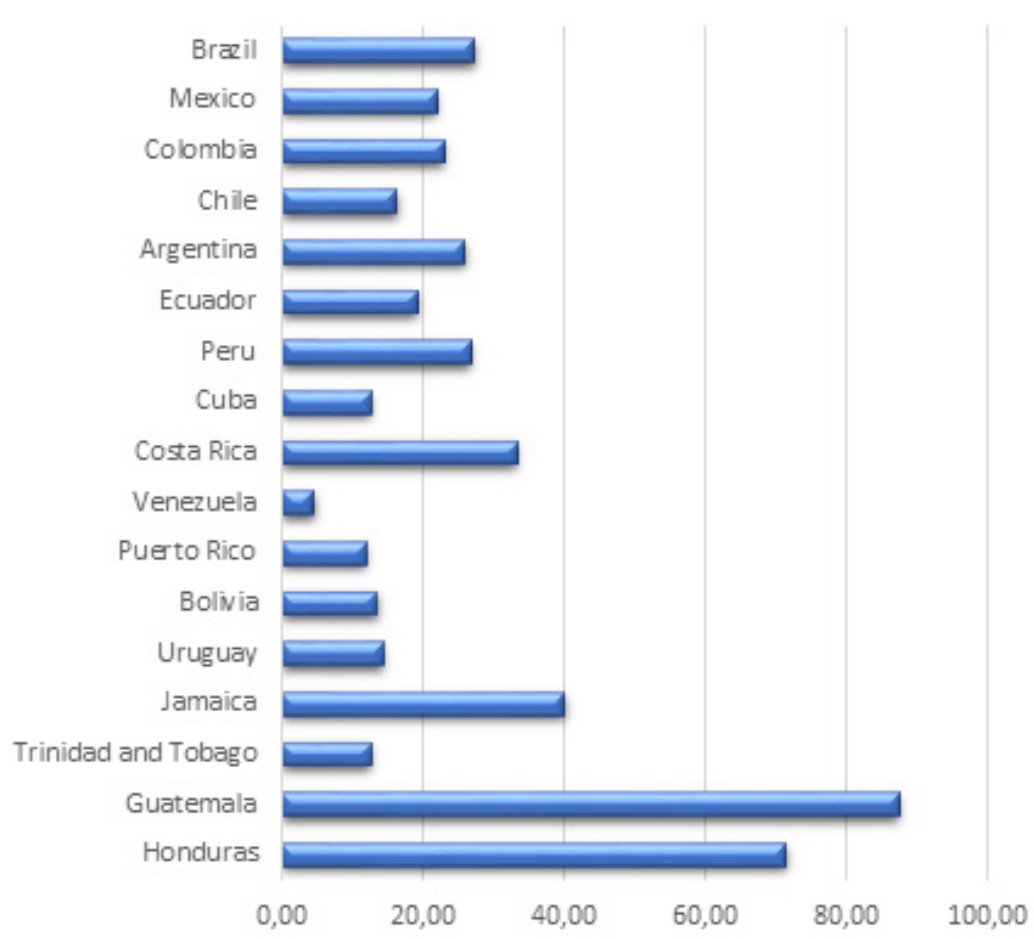

Figura 6. Distribución de las publicaciones en acceso abierto sobre ciencia abierta en América Latina. Elaboración propia.

ceso de producción científica, pareciera que el acceso abierto es uno de los grandes protagonistas del proceso ya que la mayor parte de las actividades detectadas se relacionan con su promoción.

En cuanto a las políticas públicas, el análisis de la documentación proveniente de organismos oficiales muestra que la región latinoamericana ha puesto en marcha iniciativas para favorecer la ciencia abierta reconocidas internacionalmente. En este sentido, el Global Open Policy Report (Open Policy Network, 2016) y un manual elaborado en el marco del proyecto FOSTER (Horizonte 2020), mencionan el importante esfuerzo realizado en América Latina por países como Argentina, México y Perú en materia de legislación sobre acceso abierto (Bezjak et al., 2018).

Los resultados obtenidos en este trabajo nos han permitido corroborar que, además de las normativas, también se destacan las iniciativas estatales para el progresivo desarrollo de infraestructuras para el acceso abierto a publicaciones que han llevado a la creación de Repositorios Nacionales en Argentina, Brasil, Chile, Colombia, México y Perú, algunos de creación muy reciente (2018-2019).

Muy ligadas al acceso abierto también, se han detectado acciones de planificación de apertura de datos de investigación en convocatorias de financiamiento de proyectos en Argentina (a nivel nacional) y en Brasil (a nivel federal) así como iniciativas aún en discusión para la promoción de políticas de apertura de datos de investigación en Chile.

En tal sentido, adicionalmente a las actividades relacionadas con acceso abierto a las publicaciones o a los datos de investigación, en países como Argentina, Colombia y México se han comenzado a formular acciones para la articulación de políticas públicas de promoción de ciencia abierta en un sentido más amplio. Entre ellas, se destacan la iniciativa desarrollada por Colombia en cuanto a la promoción de recursos educativos abiertos que ha llevado al desarrollo de una estrategia nacional y las actividades de sensibilización de la comunidad científica y extra-científica en cuanto a la diversidad de prácticas de ciencia abierta y ciudadana existentes y sus beneficios y desafíos encaradas por Argentina, que prevén incluir el diseño de planes de formación y construcción de capacidades.

Como resultado de este panorama, es comprensible que algunos autores señalen que el acceso abierto es una práctica conocida y relativamente utilizada entre los científicos latinoamericanos pero que, sin embargo, otras prácticas como la ciencia ciudadana, la evaluación abierta de pares o el hardware libre para la ciencia, constituyen todavía un nicho para unos pocos iniciados y son prácticamente desconocidas para el resto de los científicos (Fressoli y Arza, 2018).

Para ello, es necesario que los países de la región avancen hacia la formulación e implementación de políticas estatales de promoción sistemática y coordinada de toda la diversidad de prácticas de ciencia abierta. Múltiples desafíos se presentan para su concreción. Entre otros, sería estratégica la 
sensibilización incremental y la formación básica y avanzada en materia de ciencia abierta de los hacedores y gestores de políticas científicas y tecnológicas de las instituciones de financiamiento y ejecución de la investigación en cada uno de los países de la región para la elaboración y puesta en marcha de políticas organizacionales que fomenten la ciencia abierta referidas tanto al acceso abierto a publicaciones y datos de investigación en las instituciones que aún no han comenzado a trabajar en la cuestión como a la promoción de la ciencia ciudadana, la evaluación abierta y otras prácticas de conocimiento abierto. Tal sensibilización y formación debería estar orientada fundamentalmente a hacedores y gestores de políticas científicas y tecnológicas de universidades y entidades públicas de investigación, pero también de organismos de coordinación de las instituciones de educación superior como consejos de rectores y otro tipo de instituciones. Complementariamente, sería estratégica la realización de acciones sistemáticas de difusión y discusión, entre las comunidades científicas, de las "buenas prácticas" de conocimiento abierto y colaborativo desarrolladas y en curso en los distintos países de la región, sus beneficios y potencialidades futuras. También resultaría clave la capacitación de los diversos actores involucrados en la producción de conocimiento científico en torno a metodologías, herramientas y plataformas que faciliten la apertura de los procesos de investigación, orientada especialmente -aunque no en forma exclusiva- a investigadores, becarios de investigación y estudiantes doctorales, editores científicos y bibliotecarios. Asimismo, sería preciso avanzar en la revisión de los sistemas de evaluación de la investigación a fin de que valoren positivamente las contribuciones de los investigadores en ciencia abierta e incorporen crecientemente prácticas de evaluación por pares abierta.

En cuanto al análisis de las publicaciones sobre ciencia abierta, el uso de la base de datos internacional SCOPUS ha permitido recuperar un importante volumen de produccion regional ya que, como han puesto de manifiesto algunos autores, su cobertura en Latinoamerica es mucho mas amplia que Web of Science (Santa y Herrero-Solana, 2010; Miguel, 2011).

Los resultados sobre producción científica en Latinoamérica, muestran que es un tema de interés creciente y muy actual ya que un $66 \%$ de los documentos sobre este tema se han publicado en los últimos 5 años.

Asimismo, se ha detectado un incremento constante del aporte de la región a la producción del mundo que actualmente representa el 6\%. Algo similar ocurre con la producción de España y Portugal sobre ciencia abierta cuyos perfiles de actividad se muestran muy similares a los de Latinoamérica (De Filippo y otros, 2019). Aunque todos los países cuentan con producción científica sobre el tema, los grandes países productores son los que, además, presentan mayor volumen de documentos sobre esta temática.
El análisis de las áreas temáticas en los que concentra la mayor producción científica muestra que destacan Computer Science y Social Sciences, lo que resulta lógico ya que muchas publicaciones estudian aspectos técnicos relacionados con el acceso abierto, la interoperabilidad de datos y cuestiones anexas. Asimismo, también cobran relevancia las publicaciones sobre temas vinculados con el impacto de la ciencia abierta en la sociedad, la participación ciudadana, la comunicación, etcétera. Esta especialización temática de los documentos sobre ciencia abierta es también coincidente con la detectada en otros estudios previos realizados en diferentes contextos (Bautista-Puig y otros., 2019; De Filippo y otros, 2019).

Se ha podido comprobar que los términos más usados en las publicaciones son Open Acess, Open Data y Open Innovation pero también adquieren gran relevancia otros como Participatory Research y Community. Esto se relaciona directamente con las áreas temáticas mencionadas como las de mayor volumen documental y muestra la dualidad de la producción en las dos grandes esferas detectadas.

Si bien cabría esperar que una parte importante de la producción sobre ciencia abierta se divulgara en revistas de acceso abierto, el porcentaje de documentos de Latinoamérica sobre Open Science en Open Access es menor de lo esperado. Curiosamente son los pequeños productores (en términos de volumen documental) los que presentan mayores proporciones de documentos en abierto como Guatemala y Honduras que superan el 50\%. Entre los grandes productores, en cambio, el porcentaje de publicaciones en abierto no alcanza el $30 \%$.

Con relación a las limitaciones del trabajo, caben dos aclaraciones. Primero, que el estudio se ha centrado en el análisis de las iniciativas de política pública desarrolladas por organismos estatales con ámbito de aplicación nacional o federal. Han quedado, por tanto, explícitamente excluidas las políticas e iniciativas puestas en marcha por instituciones de educación superior o por entidades de la sociedad civil vinculadas a la temática en América Latina. Se trata de un recorte analítico en cuanto al tipo de iniciativas estudiadas que asume que el marco general de las políticas públicas desplegadas por las entidades gubernamentales es un importante reflejo de los principales lineamientos estratégicos desplegados por cada uno de los países de la región. Sin dudas, un panorama sistemático de las políticas formuladas y ejecutadas en ellos a nivel de las universidades y las entidades públicas de investigación y las iniciativas -algunas de ellas pioneras y muy destacadas, otras en pleno desarrollo- puestas en práctica desde las organizaciones de la sociedad civil resultará fundamental para la comprensión de la evolución del compromiso de la región con la promoción de la ciencia abierta.

Segundo, que existen numerosas prácticas "invisibles" de ciencia abierta que no son susceptibles de ser captadas a través de un análisis cuantitativo de la producción científica. 
Entre ellas se encuentran nuevos proyectos de ciencia ciudadana que se suman a otros más consolidados en la región, el desarrollo de software de código abierto y de prometedores proyectos de hardware científico abierto, e incipientes experiencias de evaluación abierta de trabajos de investigación -donde se registran hasta el momento sólo una revista argentina y tres brasileras con revisión de pares abierta-, entre otras muchas prácticas que se están desarrollando progresivamente en la región y que ponen de manifiesto un aspecto valioso de la dinámica de la ciencia abierta y colaborativa. La experiencia acumulada en los dos últimos años por el Ciclo de Charlas y Talleres sobre Ciencia Abierta y Ciudadana de Argentina viene dando cuenta de la riqueza y diversidad de prácticas ya existentes y permite suponer su coexistencia con realidades similares en varios países de la región.

\section{Conclusiones}

Los resultados obtenidos nos llevan a plantear que en Latinoamérica las iniciativas para el desarrollo y promoción de la ciencia abierta resultan recientes -se despliegan a lo largo de la última década- y que aún las experiencias más destacadas -que suceden en los países pioneros y más activos de la región en esta cuestión- se concentran fundamentalmente en la promoción del acceso abierto, sus infraestructuras y normativas.

Esto se evidencia con claridad en las principales políticas estatales de ciencia abierta de los países latinoamericanos, en cuya evolución se observa una importante influencia de los lineamientos de políticas promovidos por la Comisión Europea, la Organización para la Cooperación y el Desarrollo Económicos (OCDE) y otros organismos de los países centrales que fomentan el aumento de la transparencia gubernamental y la participación pública. Si bien un análisis comparado de las políticas de ciencia abierta en Europa y en Latinoamérica que aborde en profundidad la influencia y repercusión de unas sobre otras y las diferentes direcciones y dinámicas que presentan en cada región excede los alcances de este trabajo, queda abierto para futuras exploraciones.

También se evidencia la concentración de la promoción del acceso abierto en el contenido de las publicaciones científicas producidas en Latinoamérica sobre la temática. Los datos de investigación abiertos parecen estar abriéndose camino en los últimos años en las publicaciones especializadas, aunque aún son incipientes las políticas públicas para su regulación y fomento.

El contenido de las publicaciones muestra también que existe una preocupación desde el ámbito académico por temas relacionados con el impacto social, la participación ciudadana y aspectos que van más allá del acceso abierto; y en este sentido queda abierta también la exploración de mecanismos alternativos al análisis cienciométrico clásico para visibilizar otro tipo de prácticas de ciencia abierta que tienen creciente presencia en los países latinoamericanos. Un reflejo de esta preocupación ha sido la firma reciente de la Declaración de Panamá (2018) que aboga por una ciencia más transparente, colaborativa y participativa y problematiza su rol para alcanzar una educación y una ciencia de calidad en la región.

La ciencia abierta implica un cambio de paradigma que está afectando los modos de producir, difundir, evaluar y comunicar la ciencia en todas las regiones del mundo, y es de esperar que progresivamente más actores de América Latina (gobiernos, académicos, organizaciones de la sociedad civil, editores, bibliotecarios y otros) tomen la iniciativa para promover los cambios institucionales y culturales que presten atención y valoren las prácticas de ciencia abierta y colaborativa que ya se están promoviendo en la región y generen las condiciones para multiplicarlas en aras de lograr una sociedad más inclusiva.

\section{Agradecimientos}

Los aspectos metodológicos cienciométricos desarrollados en este trabajo han sido realizados en el marco del Proyecto "Detección de nuevos frentes de investigación e innovación. Análisis de los flujos de conocimiento entre el ambito científico, la industria y la sociedad" (REF: CSO2014-51916-C21-R) financiado por el Ministerio Español de Economía y Competitividad (MINECO).

Las autoras agradecen los valiosos comentarios y sugerencias realizados por Mariano Fressoli, investigador del CONICET en el CENIT, Argentina, y el material documental provisto por Paola Azrilevich, secretaria ejecutiva del Sistema Nacional de Repositorios Digitales del MECCYT, Argentina. Asimismo, agradecen los valiosos comentarios de dos revisores anónimos.

\section{Nota al final}

\begin{abstract}
Una interesante herramienta para la identificar y analizar estas iniciativas a nivel internacional es Políticas MELIBEA, un directorio y estimador de políticas en favor del acceso abierto a la producción científica. Está centrada en instituciones académicas o de investigación y entidades financiadoras e incluye algunos países latinoamericanos. Ha sido desarrollada en 2018 por la Universidad de Barcelona y el Consejo de Investigaciones Científicas y Técnicas (CSIC) y está disponible en https://www.accesoabierto.net/politicas/.
\end{abstract}

\section{Referencias}

Actis, G. y Carlino, F. (2017). Plan de Gestión de Datos en CONICET: análisis, experiencia y desafíos. La Plata: VII Conferencia Internacional sobre Bibliotecas y Repositorios Digitales de América Latina (BIREDIAL-ISTEC'17) y XII Simposio Internacional de Bibliotecas Digitales (SIBD'17), 215-231. Recuperado de: http://sedici.unlp.edu.ar/ handle/10915/63588

Anglada, L., y Abadal, E. (2018). ¿Qué es la Ciencia Abierta? Anuario 
ThinkEPI, 12, 292-298. https://doi.org/10.3145/thinkepi.2018.43

Bautista-Puig, N., De Filippo, D., Mauleón, y E., Sanz-Casado, E. (2019) Scientific landscape of Citizen Science publications: dynamics, content and presence in social media. Publications: Dynamics, Content and Presence in Social Media, 7(1). https://doi.org/10.3390/ publications 7010012

Bezjak, S., Conzett, P., Fernandes, P.L., Görögh, E., Helbig, K., Kramer, B., Labastida, I, Niemeyer, K., Psomopoulos, F., Ross-Hellauer, T., Schneider, R., Tennant, J., Verbakel, E., Clyburne-Sherin, A., Brinken, H., y Heller, L. (2017). Manual de capacitación sobre Ciencia Abierta. Foster. https://doi.org/10.5281/zenodo.2583101

COLCIENCIAS: Departamento Administrativo de Ciencia, Tecnología e Innovación (2018). Lineamientos para una política de ciencia abierta en Colombia. Bogotá: COLCIENCIAS. Recuperado de: https:// www.colciencias.gov.co/sites/default/files/ckeditor_files/Lineamientos\%20ciencia\%20abierta\%2017-dic-2018-doc.pdf

CONACYT: Consejo Nacional de Ciencia y Tecnología de México (2014). Lineamientos generales para el Repositorio Nacional y los Repositorios Institucionales. Recuperado de: https://www.repositorionacionalcti.mx/docs/Lineamientos_generales.pdf

CONACYT: Consejo Nacional de Ciencia y Tecnología de México (2017a). Lineamientos generales de Ciencia Abierta. Recuperado de: http://www.siicyt.gob.mx/index.php/normatividad/conacyt-normatividad/programas-vigentes-normatividad/lineamientos/lineamientos-generales-de-ciencia-abierta/4707-lineamientos-generales-de-ciencia-abierta/file

CONACYT: Consejo Nacional de Ciencia y Tecnología de México (2017b). Lineamientos jurídicos de Ciencia Abierta. Recuperado de: http://www.siicyt.gob.mx/index.php/normatividad/conacyt-normatividad/programas-vigentes-normatividad/lineamientos/lineamientos-juridicos-de-ciencia-abierta/3828-lineamientos-juridicos-de-ciencia-abierta/file

CONCYTEC: Consejo Nacional de Ciencia, Tecnología e Innovación Tecnológica de Perú (2017). Directrices para el procesamiento de información en los Repositorios Institucionales. Recuperado de: https://portal.concytec.gob.pe/images/documentos/alicia/directrices_repositorio.pdf

CONCYTEC: Consejo Nacional de Ciencia, Tecnología e Innovación Tecnológica de Perú (2019). Repositorio Nacional Digital de Acceso Abierto. Recuperado de: http://portal.concytec.gob.pe/index.php/ informacion-cti/alicia

Congreso de la República de Perú (2013). Ley 30.035 que regula el Repositorio Nacional Digital de Ciencia, Tecnología e Innovación de Acceso Abierto. Recuperado de: https://portal.concytec.gob. pe/images/stories/images2013/portal/areas-institucion/dsic/ ley-30035.pdf

CONICYT: Consejo Nacional de Investigación Científica y Tecnológica de Chile (2014). Propuesta de Política de datos abiertos. Recuperado de: http://datoscientificos.cl/politica

CONICYT: Consejo Nacional de Investigación Científica y Tecnológica de Chile (2019). Repositorio CONICYT. Recuperado de: http://repositorio.conicyt.cl/

da Silva, P. (2017). As Políticas de Open Data em Portugal: análise da sua implementação e impacto. [Tesina de la Maestría en Ciencias de la Información]. Recuperado de http://hdl.handle.net/10316/85604

Declaración de Panamá para la Ciencia Abierta (2018). Recuperado de https://hiperderecho.org/wp-content/uploads/2018/11/declaracion_panama_ciencia_abierta.pdf
De Filippo, D., Silva, P., y Borges, M.M. (2019). Caracterización de las publicaciones de España y Portugal sobre open science y análisis de su presencia en las redes sociales. Revista española de documentación científica, 42 (2). https//doi.org/10.3989/redc.2019.2.1580

España. Ministerio de Economía, Industria y Competitividad, Secretaría de Investigación, Desarrollo e Innovación (2017). Plan Estatal de Investigación Científica y Técnica y de Innovación 2017-2020. Recuperado de: http://www.idi.mineco.gob.es/stfls/MICINN/Prensa/ FICHEROS/2018/PlanEstatalIDI.pdf

European Commission (2016). Open innovation, Open Science, open to the world. A vision for Europe. Brussels: European Commission, Directorate-General for Research and Innovation. Recuperado de: http:// bookshop.europa.eu/en/open-innovation-open-science-open-totheworld- pbKI0476263/

European Commission (2017). Open Science Monitor. Brussels: European Commission, Directorate-General for Research and Innovation. Recuperado de: http://ec.europa.eu/research/openscience/index. cfm?pg=about\&section=monitor

European Union (2013). Regulamento (UE) No 1291/2013 do Parlamento Europeu e do Conselho de 11 de dezembro de 2013. Jornal Oficial da União Europeia. Recuperado de: http://ec.europa.eu/ research/participants/data/ref/h2020/legal_basis/fp/h2020-eu -establact_pt.pdf

FAPESP: Fundación de Apoyo a la Investigación del Estado de Sāo Paulo (2017). FAPESP anuncia novas normas para submissão de Projetos Temáticos. Plano de gestão de dados. Recuperado de: http:// www.fapesp.br/11273

FAPESP: Fundación de Apoyo a la Investigación del Estado de Sāo Paulo (2019). Portaria Conselho Técnico-Administrativo FAPESP 01/2019 Política para Acesso Aberto às Publicações Resultantes de Auxílios e Bolsas FAPESP. Recuperado de: http://www.fapesp. $\mathrm{br} / 12632$

Fernández, M.L. (2019). Entrevista personal con María Laura Fernández, Subsecretaria Ejecutiva de la Agencia Nacional de Investigación e Innovación (ANII) de Uruguay, 29 de marzo de 2019.

Finland. The Ministry of Education and Culture's Open Science and Research Initiative 2014-2017 (2014). The Open Science and Research Roadmap. Reports of the Ministry of Education and Culture. Finland, 2014:21. Recuperado de: https://openscience.fi/documents/14273/0/ Open+Science+and+Research+Roadmap+2014-2017/e8eb7704-8ea748bb-92e6-c6c954d4a2f2

Follett, R., y Strezov, V. (2015) An Analysis of Citizen Science Based Research: Usage and Publication Patterns. PLOS ONE, 10(11): https:// doi.org/10.1371/journal.pone.0143687

Forsström, P., y Haataja, J. (2016). Open Science as an Instrument for Effective Research. Recuperado de: http://ojs.tsv.fi/index.php/ signum/article/viewFile/58741/20303

Fosci, M., Richens, E. y R. Johnson (2019). Insights into European research funder Open policies and practices. SPARC Europe. Recuperado de: https://zenodo.org/record/3401278\#.Xa7h6y3SHEo

FOSTER (2016). Open Science Definition. Recuperado de: https:// www.fosteropenscience.eu/foster-taxonomy/open-science-definition

Fressoli, M., y Arza, V. (2018). Los desafíos que enfrentan las prácticas de ciencia abierta, Teknokultura. Revista de Cultura Digital y Movimientos Sociales, 15(2), 429-48

Honorable Congreso de la Nación Argentina (2013). Ley 26899. 
Recuperado de: https://www.argentina.gob.ar/normativa/nacional/ ley-26899-223459

IBICT: Instituto Brasileiro de Informacāo em Ciencia e Tecnologia (2019). Portal brasileiro de publicações científicas em acesso aberto. Recuperado de: http://oasisbr.ibict.br/vufind/

IAA: Instituto Antártico Argentino (2018). Políticas del Repositorio Digital Institucional del Instituto Antártico Argentino. Acceso Abierto a Publicaciones y Datos Primarios. Versión 1.7. Ciudad Autónoma de Buenos Aires: IAA.

Kullenberg, C.; Kasperowski, D. (2016). What is citizen science? - A scientometric meta-analysis. PLOS ONE, 11, e0147152

LA Referencia: Red de Repositorios de Acceso Abierto a la Ciencia (2019). Compartiendo y dando visibilidad a la producción científica en América Latina. Recuperado de: http://www.lareferencia.info/es/ institucional/quienes-somos

Leite, C. (2015). Transparencia e acceso abierto a la información en Brasil. VIII Coloquio sobre Brecha Digital. Madrid: Universidad Carlos III de Madrid. Recuperado de http://portal.uc3m.es/portal/ page/portal/inst_docum_gest_info_agustin_millares/VIII_Brecha_ digital/Programa/Cecilia-PPT\%20Cec\%EDlia\%20Madrid\%20 a\%E7\%F5es\%20de\%20governo\%20e\%20cient\%EDfica.pdf

LERU: League of European Research Universities (2018). Open Science and its role in universities: A roadmap for cultural change. Advice paper 24. Recuperado de: https://www.leru.org/publications/ openscience-and-its-role-in-universities-a-roadmap-for-cultural-change

MECCYT: Ministerio de Educación, Cultura, Ciencia y Tecnología de Argentina (2011). Sistema Nacional de Repositorios Digitales. Recuperado de: http://repositoriosdigitales.mincyt.gob.ar/vufind/

MECCYT: Ministerio de Educación, Cultura, Ciencia y Tecnología de Argentina (2016). Resolución E 753/2016. Recuperado de: https://www.argentina.gob.ar/normativa/nacional/resolución-753-2016-267833

MECCYT: Ministerio de Educación, Cultura, Ciencia y Tecnología de Argentina (2017a). Resolución E 640/2017. Recuperado de: https://www.argentina.gob.ar/normativa/nacional/resolución-640-2017-277216

MECCYT: Ministerio de Educación, Cultura, Ciencia y Tecnología de Argentina (2017b). Portal de Información de Ciencia y Tecnología Argentino. Recuperado de: https://datos.mincyt.gob.ar

MECCYT: Ministerio de Educación, Cultura, Ciencia y Tecnología de Argentina (2019). Ciencia Abierta y Ciudadana. Recuperado de: https://www.argentina.gob.ar/ciencia/sact/ciencia-abierta-y-ciudadana

MEXICO (2014). Decreto por el que se reforman y adicionan diversas disposiciones de la Ley de Ciencia y Tecnología, de la Ley General de Educación y de la Ley Orgánica del Consejo Nacional de Ciencia y Tecnología. Diario Oficial de la Federación. Recuperado de: https://www.dof.gob.mx/nota_detalle.php?codigo=5345503\&fecha $=20 / 05 / 2014$

Miguel, S. (2011). Revistas y producción científica de América Latina y el Caribe: su visibilidad en SciELO, RedALyC Y SCOPUS. Revista Interamericana de Bibliotecología, 34(2), 187-199

MinEducación: Ministerio de Educación Nacional de Colombia (2013). Estrategia REDA. Recuperado de: http://186.113.12.159/Documentacion/Estrategia_REDA.pdf

MinEducación: Ministerio de Educación Nacional de Colombia (2019). Sistema Nacional de Acceso Abierto al Conocimiento, SNAAC. Recu- perado de: http://snaac.renata.edu.co:8080/web/guest/estrategia-snaac

Netherlands. Dutch Ministry of Education, Culture and Science (2017). National Plan Open Science. https.//doi.org/10.4233/uuid:9e9fa82e06c1-4d0d-9e20-5620259a6c65

OECD (2015). Making Open Science a Reality. OECD Science, Tecnology and Industy Policy Papers, 25. Paris: OECD Publications. http://doi. org/10.1787/5jrs2f963zs1-en

Open Policy Network (2016). Global Open Policy Report 2016. Recuperado de: https://creativecommons.org/wp-content/ uploads/2016/12/StateofOpenPolicyFullReport_FINAL-1-1-1-1.pdf

OSI. (2019) Policy Perspective: Plan S \& the quest for global open Access. Recuperado de: http://osiglobal.org/2019/03/13/osi-policy-perspective-plan-s-the-quest-for-globalopen-access

Oszlak, O., y O'Donnell, G. (1981). Estado y políticas estatales en América Latina: hacia una estrategia de investigación. Buenos Aires: Centro de Estudios de Estado y Sociedad (CEDES)

Palma Peña, J.M. (2019). "El Plan 'S' en la perspectiva de la bibliotecología de América Latina: análisis y propuestas para la implementación e innovación". Trabajo presentado en: IFLA WLIC 2019. Atenas, Grecia. Recuperado de: http://library.ifla.org/2455/1/138-palma-es. pdf

Portugal. Ministério da Ciência, Tecnologia e Ensino Superior (MCTES) (2016). Ciência Aberta, Conhecimento para todos: Princípios orientadores. Recuperado de: http://www.portugal.gov.pt/media/18506199/ 20160210-mctes-ciencia-aberta.pdf

Presidencia del Consejo de Ministros de Perú (2015). Decreto supremo N $006-2015-p c m$. Recuperado de: http://www2.congreso.gob.pe/ sicr/cendocbib/con5_uibd.nsf/8B77FD28349C12B70525826C0075CBOC/\$FILE/4_Aprueban_Reglamento_de_la_Ley_30035.pdf

Research Information Network (2010). Open science case studies. Recuperado de: http://www.rin.ac.uk/system/files/attachments/ RINews_Issue_11_0.pdf

Ramírez, P.A., y Samoilovich, D. (2018). Ciencia abierta. Reporte para tomadores de decisiones. París y Montevideo: Organización de las Naciones Unidas para la Educación, la Ciencia y la Cultura (UNESCO) y Oficina Regional de Ciencias de la UNESCO para América Latina y el Caribe (UNESCO Montevideo)

Santa, S., y Herrero-Solana, V. (2010). Cobertura de la ciencia de América Latina y el Caribe en SCOPUS vs. Web of Science. Investigación bibliotecológica, 24(52), 13-27

Santos, P. X. (Coord.), Almeida, B. de Araújo, Elias, F., Motta, M. L. da, Guanaes, P., Jorge, V. de A., Henning, P., y G. Oliveira (2017) Livro Verde - Ciência aberta e dados abertos: mapeamento e análise de políticas, infraestruturas e estratégias em perspectiva nacional e internacional. Río de Janeiro: Fundação Oswaldo Cruz. Recuperado de: https://www. arca.fiocruz.br/bitstream/icict/24117/2/Livro-Verde-07-06-2018.pdf

Senado Federal del Brasil (2011) Projeto de Lei do Senado n 96, de 2011. Recuperado de: https://www25.senado.leg.br/web/atividade/ materias/-/materia/101006

STEPS América Latina (2016). Ciencia abierta, colaborativa y alternativa: un camino para resolver problemas de desarrollo. Recuperado de: http://stepsamericalatina.org/

Vicente-Saez, R., y Martinez-Fuentes, S. (2018). Open Science Now: A Systematic Literature Review for an Integrated Definition. Journal of Business Research, January 2018. https://doi.org/10.1016/j. jbusres.2017.12.043 


\section{CV}

Daniela De Filippo. Licenciada en Comunicación (Universidad Nacional de Quilmes, Argentina), Máster en Comunicación y Cultura de la Ciencia (Universidad de Salamanca, España) y Doctora en Biblioteconomía y Documentación (Universidad Carlos III de Madrid, España). Su principal área de investigación es la cienciometría y se ha especializado en el desarrollo y la aplicación de indicadores para el estudio de la actividad científica y tecnológica. En los últimos años ha comenzado a investigar sobre nuevas métricas para la evaluación científica con especial énfasis en el entorno de la ciencia abierta. Ha trabajado en centros de investigación de Argentina, Suiza y España y, desde 2011, es miembro del Laboratorio de Estudios Métricos de la Información de la Universidad Carlos III de Madrid. Actualmente es, además, investigadora en el Instituto INAECU sobre evaluación de la ciencia y la universidad (UAM-UC3M) y ha obtenido un contrato "Ramón y Cajal" para incorporarse al departamento de Biblioteconomía y Documentación de la Universidad Carlos III de Madrid. https://www. researchgate.net/profile/Daniela_De_Filippo2
María Guillermina D`Onofrio. Licenciada en Sociología y Máster en Política y Gestión de la Ciencia y la Tecnología (Universidad de Buenos Aires, Argentina) y candidata a Doctora en Ciencias Sociales (Facultad Latinoamericana de Ciencias Sociales). Desde 2016 se desempeña como Directora Nacional de Programas y Proyectos en el Ministerio de Educación, Cultura, Ciencia y Tecnología (MECCYT) de Argentina, donde ha estado trabajando en planificación y gestión de la política científica, generación y análisis de información científica y evaluación científica y tecnológica hace más de quince años. Está a cargo del Programa de Datos de Abiertos de Ciencia y Tecnología, del Sistema de Información de Ciencia y Tecnología Argentino (SICYTAR) y de la Agenda de Género en Ciencia y Tecnología del MECCYT. Su principal área de investigación es la sociología de la ciencia y se ha especializado en el desarrollo y la aplicación de indicadores de ciencia y tecnología con especial énfasis en el estudio de las carreras científicas y en los impactos de los sistemas de evaluación de la investigación. https://ar.linkedin. com/in/maría-guillermina-d-onofrio-8968728 осм Observatorio de Cibermedios

\section{https://observatoriocibermedios.upf.edu/}

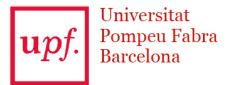

Departamento de Comunicación
Grupo DigiDoc
El Observatorio de Cibermedios es una producción del Grupo de Investigación en Documentación Digital y Comunicación Interactiva (DigiDoc) del Departamento de Comunicación de la Universitat Pompeu Fabra.

El Observatorio de Cibermedios (OCM) forma parte del proyecto del Plan Nacional "Creación y contenido interactivo en la comunicación de información audiovisual: audiencias, diseño, sistemas y formatos". CSO2015-64955-C4-2-R (MINECO/ FEDER), Ministerio de Economía y Competitividad (España). 